\title{
Image of the month: Placental transmogrification of the lung: a rare cause of cystic lung disease
}

\author{
Authors: Dalia Mudawi, ${ }^{\mathrm{A}}$ Rabia Touqeer, ${ }^{\mathrm{B}}$ Angeles Montero-Fernandez, ${ }^{\mathrm{C}}$ Melanie Greaves ${ }^{\mathrm{D}}$ and \\ George A Margaritopoulos ${ }^{\mathrm{E}}$
}

KEYWORDS: Placental transmogrification, cyst, benign, respiratory

DOI: $10.7861 /$ clinmed.2020-0584

\section{Case presentation}

A 40-year-old woman, who was an ex-smoker, presented with a 2-month history of dry cough, and sensation of fluid movement within the thorax. Past medical history included only liver haemangiomas, for which she was under surveillance. Computed tomography (CT) of the thorax, axial image of lung and soft tissue, demonstrated a large thin-walled fluid collection in the region of the right major fissure. The collection extended into the right paracardiac and posterior mediastinal regions. Lung adjacent to this was atelectatic, but the remaining parenchyma was normal (Fig 1). The patient underwent further investigation under the cardiothoracic team with right diagnostic videoassisted thoracoscopic surgery. This showed three thin walled cystic lesions, containing both fluid and air, arising from the lung parenchyma. The fluid was aspirated and analysis revealed it to be predominantly lymphocytic, comprising small lymphocytes and foamy macrophages. No organisms, ova or parasites were seen. Routine blood tests, blood-borne virus screen and autoimmune panel were unremarkable. The patient mentioned recent travel to Africa, subsequently infectious/tropical diseases were ruled out after performing the appropriate laboratory tests.

A decision was made with the patient to proceed to surgical excision of the cysts due to risks associated with possible complications of, for example, pneumothorax. The patient made excellent recovery post-operatively, and at 3-month follow-up was found to be asymptomatic with normal lung function with no evidence of recurrent disease on repeat CT of the thorax.

Authors: A respiratory registrar, Manchester University NHS Foundation Trust, Manchester, UK; ${ }^{B}$ respiratory senior house officer, Manchester University NHS Foundation Trust, Manchester, UK; ${ }^{C}$ consultant histopathologist, Manchester University NHS Foundation Trust, Manchester, UK; ${ }^{\mathrm{D}}$ consultant radiologist, Manchester University NHS Foundation Trust, Manchester, UK; ${ }^{\mathrm{E}}$ Consultant respiratory physician, Manchester University NHS Foundation Trust, Manchester, UK and The University of Manchester, Manchester, UK

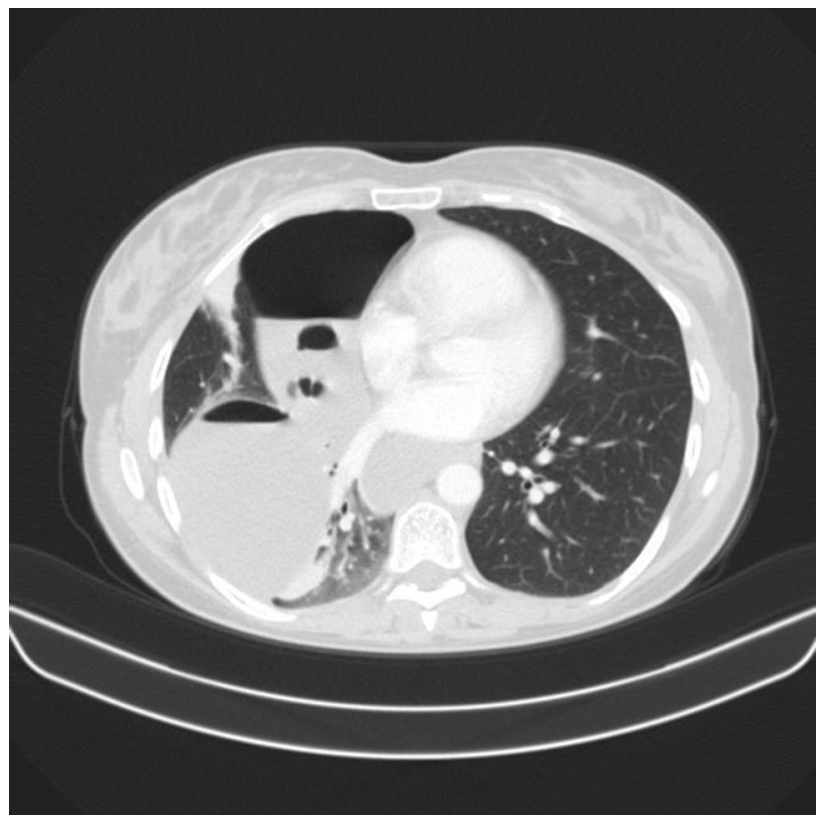

Fig 1. Computed tomography of the thorax demonstrating a large thin-walled fluid collection in the region of the right major fissure. The collection extends into the right paracardiac and posterior mediastinal regions. Lung adjacent to this is atelectatic, but the remaining parenchyma is normal.

Microscopic examination of the excised cystic wall fragments demonstrated fibrous and chronic inflammatory tissue with a focal nodular architecture and prominent lymphoid aggregates (Fig 2). There were prominent blood vessels and lymphatic channels. The cysts were focally lined by bland-looking epithelial cells. Immunohistochemistry showed patchy positivity for D240 and CD34 highlighting vascular and lymphatic spaces. AE1/3 and TTF-1 were positive in pneumocytes. CD10 showed focal stromal positivity. The findings are in keeping with placental transmogrification of the lung (PTL).

\section{Discussion}

PTL is a rare benign disease, first described in 1979. ${ }^{1}$ A recent literature review identified only 34 cases. $^{2}$ PTL has no placental 


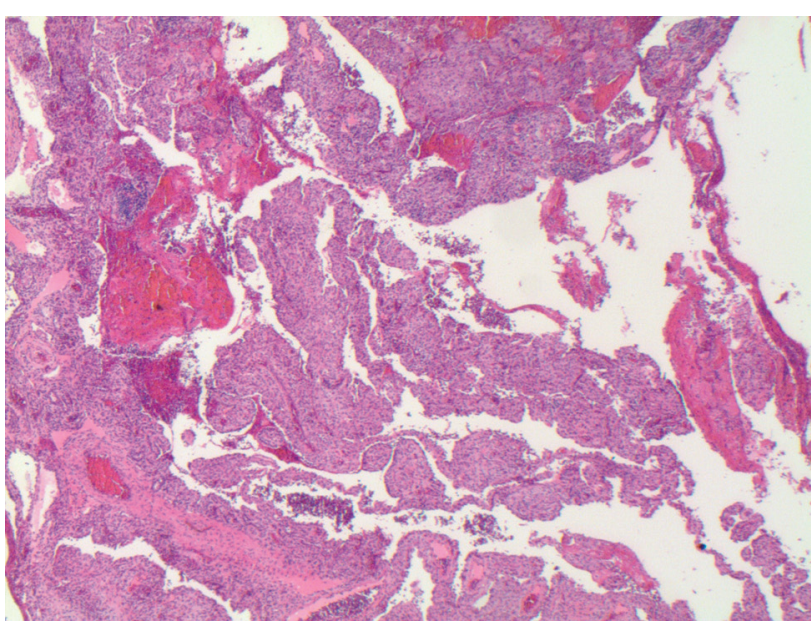

Fig 2. Haematoxylin and eosin stain of cystic wall fragments demonstrating fibrous and chronic inflammatory tissue with a focal nodular architecture and prominent lymphoid aggregates.

properties but morphologically resembles immature placental tissue. The disease predominantly affects men between 20 to 50 years of age. Patients are frequently asymptomatic, but may present with dyspnoea, chest pain, pneumothorax or chronic obstructive lung disease due to bullous emphysema. ${ }^{2,3}$

The pathogenesis still remains unclear. Proposed mechanisms include lymphovascular proliferation in the setting of an emphysematous lung and congenital hamartomatous malformation. ${ }^{3,4}$ CT of the thorax typically shows bullous lesions and rarely cysts or nodules. ${ }^{5}$

Differential diagnosis includes bullous emphysema, solitary pulmonary nodules (hamartoma), intralobar pulmonary sequestration, bronchogenic cyst and cystic lung tumours. ${ }^{2,5} \mathrm{CT}$ or magnetic resonance imaging aids physicians in distinguishing these entities from PTL, but histology is required to confirm the diagnosis. ${ }^{4}$

Surgical resection is the treatment of choice, which is usually curative and successful in improving symptoms and quality of life. There was no literature report of recurrence in those in whom complete resection was achieved.

Although rare, PTL should be considered in the differential diagnosis of cystic lung lesions. Early suspicion and diagnosis could facilitate minimal resection surgery and prevent complications.

\section{References}

1 McChesney TM. Placental transmogrification of the lung: A unique case with remarkable histopathologic features. Lab Invest 1979; 40:245-6.

2 Ma DJ, Liu HS, Li SQ et al. Placental transmogrification of the lung: Case report and systematic review of the literature. Medicine (Baltimore) 2017;96:e7733.

3 Narula N, Ngua S, Sharmab D et al. Placental transmogrification of the lung associated with unilateral pleural effusion: A case report with a comprehensive review of the literature. Respiratory Medicine Case Reports 2019;26:161-4.

4 Ventura L, Gnetti L, Silini EM et al. Placental transmogrification of the lung presenting as a giant bulla associated with a pulmonary hamartoma. Ann Thorac Surg 2016;102:e61.

5 Kim JW, Park IH, Kwon W et al. Placental transmogrification of the lung. Korean J Radiol 2013;14:977-80.

Address for correspondence: Dr George A Margaritopoulos, ILD Unit, Wythenshawe Hospital, Manchester University NHS Foundation Trust, Southmoor Road, Wythenshawe, Manchester M23 9LT, UK.

Email: gmargaritop@yahoo.gr 